\title{
Corrigendum: Salicylic acid biosynthesis is enhanced and contributes to increased biotrophic pathogen resistance in Arabidopsis hybrids
}

Li Yang, Bosheng Li, Xiao-yu Zheng, Jigang Li, Mei Yang, Xinnian Dong, Guangming He, Chengcai An \& Xing Wang Deng

Nature Communications 6:7309 doi: 10.1038/ncomms8309 (2015); Published online 12 Jun 2015; Updated 12 Aug 2015

In the last paragraph of the Discussion section in this Article, the hybrid Ler \& C24 was incorrectly referred to as Ler \& Col-0. The following is the correct sentence: 'The F1 hybrids from two combinations (Col-0 \& C24 and Ler \& C24), which exhibit heterosis for biomass $^{11,14}$, show no heterosis for biotrophic bacteria defence in our study (Supplementary Fig. 11)'. 